\title{
WSCC 2018: the 2018 World Speed Chess Championship
}

Article

Accepted Version

Krabbenbos, J., van den Herik, J. and Haworth, G. (2018) WSCC 2018: the 2018 World Speed Chess Championship. ICGA Journal, 40 (3). pp. 207-210. ISSN 2468-2438 doi: https://doi.org/10.3233/ICG-190078 Available at https://centaur.reading.ac.uk/78438/

It is advisable to refer to the publisher's version if you intend to cite from the work. See Guidance on citing.

To link to this article DOI: http://dx.doi.org/10.3233/ICG-190078

Publisher: IOS Press

All outputs in CentAUR are protected by Intellectual Property Rights law, including copyright law. Copyright and IPR is retained by the creators or other copyright holders. Terms and conditions for use of this material are defined in the End User Agreement.

\section{www.reading.ac.uk/centaur}

\section{CentAUR}

Central Archive at the University of Reading

Reading's research outputs online 


\section{WSCC 2018: The World Speed Computer Chess Championship}

Jan Krabbenbos, Jaap van den Herik and Guy Haworth ${ }^{1}$

Amersfoort, the Netherlands, Leiden, the Netherlands and Reading, UK

WSCC 2018 took place on July $13^{\text {th }}$ at the Stockholmsmässan in Stockholm, Sweden and was organised by the ICGA as an opener before the 'WCSC' World Chess Software and 'WCCC' World Computer Chess Championships (Krabbenbos et al., 2018a/b). Using their WCCC hardware, five of the eight WCCC participants took part as in Table 1: the format was therefore five rounds at a tempo of $5^{\prime}+5^{\prime \prime} /$ move. The venue was part of the international conferences IJCAI, ECAI, AAMAS, ICML, ICCBR and SoCS. The main sponsor was Digital Game Technology (DGT).

This was the first time for many years that this computer chess tournament had been played alongside a large artificial intelligence conference. This resulted in large crowds around the boards and, see Fig. 1 , they were able to witness grandmaster-level play despite the blitz tempo. The high levels of stress on the operators, only one finger-slip away from a loss, was palpable.

During the player meeting, the number of games per round was discussed. There seemed to be plenty of time to do more games than the normal two per round for the five participants and it was decided to play three games per round. Looking back with the knowledge of how the games went, the necessary time for playing all the rounds was almost not enough. There were different reasons for this but the setup time for some of the programs was one of the main issues. JONNY in particular was allowed to play two consecutive games with the same colour to minimise its setup time.

Table 1

The participants in WSCC 2018 (CPW, 2018)

\begin{tabular}{lcccc}
\hline id & Program & Author(s) & State & Operator \\
\hline C & CHIRON & Ubaldo Andrea Farina & IT & U A F \\
G & GRIDGINKGO & Frank Schneider, Kai Himstedt, Rob Hyatt & DE & Frank Schneider \\
J & JONNY & Johannes Zwanzger & DE & J Z \\
K & KOMODO & Don Dailey, Mark Lefler \& Larry Kaufman & US & Erdogen Günes \\
S & SHREDDER & Stefan Meyer-Kahlen & DE & S M-K \\
\hline
\end{tabular}

The games are fully available both online and with some annotation in a pgn attached to the repository version of this report (Krabbenbos et al.., 2018c). They are listed in Table 2 with the results in Tables 3-5. Only the KOMODO-SHREDDER and CHIRON-GRIDGINKGO mini-matches were tied at 11/2 points each.

Of all the games, from the perspective of the spectators, KOMODO-CHIRON game 2 was perhaps the most spectacular. With 21. Rxg4 as in Fig. 2a, KOMODO exposed Black's king at the cost of rook for knight and pawn. By move 32w, Fig. 2b, White's king was more exposed than Black's was but by move

\footnotetext{
${ }^{1}$ Communicating author: g.haworth@ reading.ac.uk
} 
51w as in Fig. 2c, it had found shelter near its pawns from the two black rooks. Black's king was then harried nervelessly and without cease by White's RBN trio of pieces for 25 moves, first to the edge and then to the corner of irrelevance, see Fig. 2d. At this point, CHIRON lost on time a few moves before it would have lost on the board. The further playout - FRITZ14 reaches 'mate in 32m' four moves later would have added even more to a game much appreciated by the spectators.

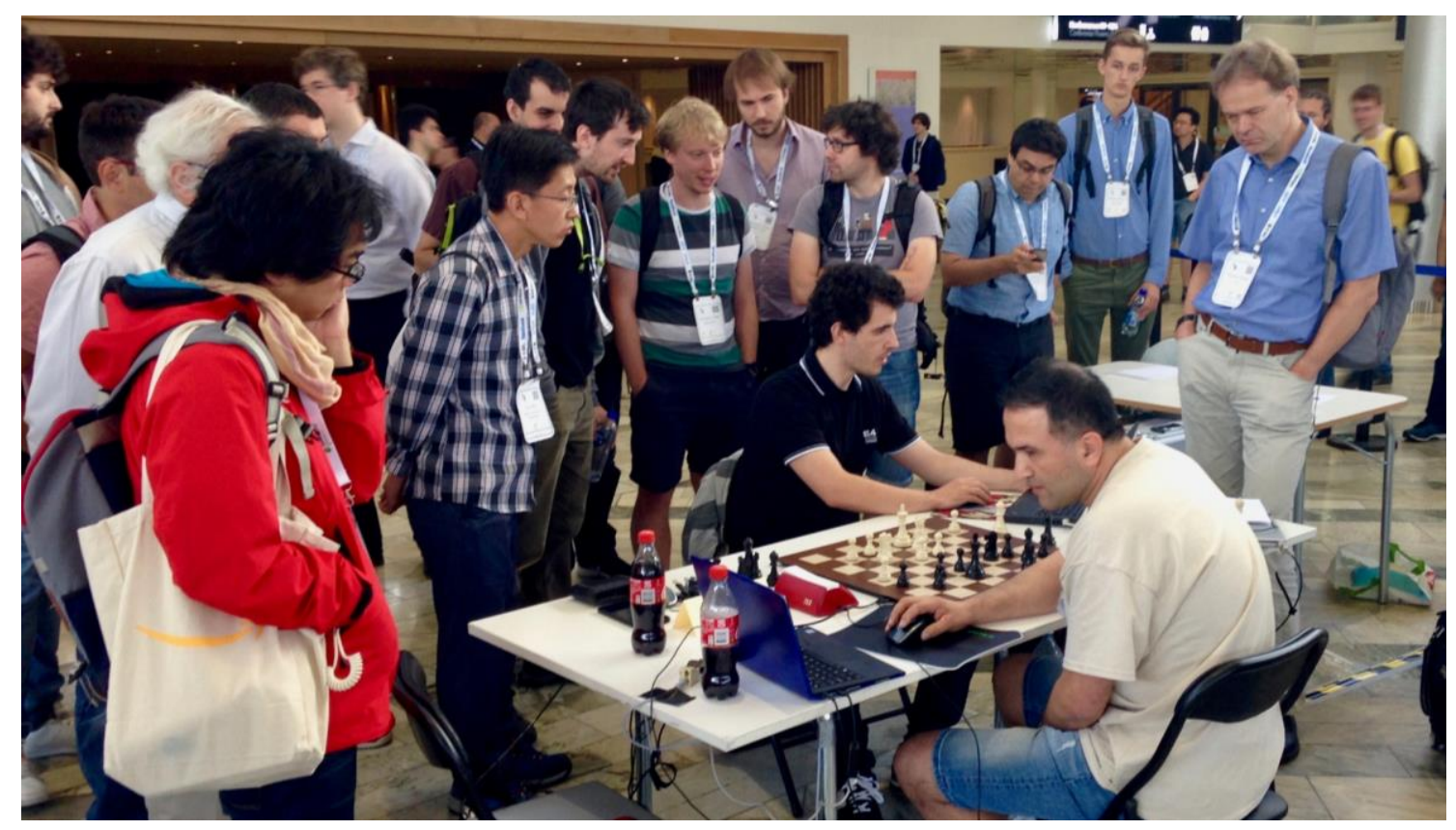

Fig. 1. Game 1.1 at position 25w: CHIRON-KomODO: operators Andrea Ubaldo (1) and Erdogen Günes.
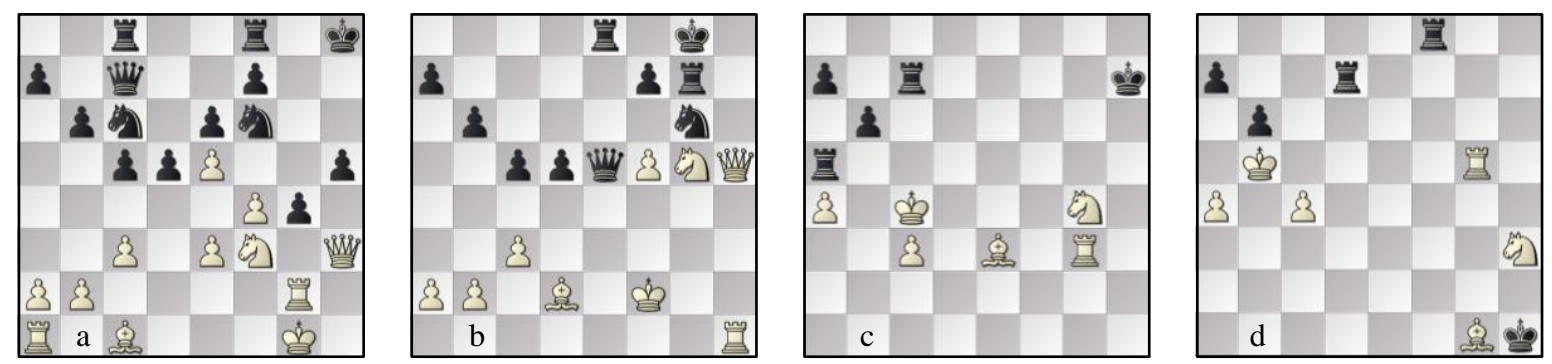

Fig. 2. Round 1.2, game 2, Komodo-CHIRON at positions (a) 21w, (b) 32w, (c) 51w and (d) $76 \mathrm{~b}$.

During the tournament, two games were lost due to operator error: round 2.1 game 7, KOMODOGRIDGINKGO and round 4.2 game 23, CHIRON-GRIDGINKGO. Such errors are never intentional and the outcome is always an unfortunate anti-climax for all concerned. This eventuality had also been discussed in the player meeting prefacing the tournament. Several other solutions were proposed but all of them seemed to have the same problem, that of determining how to solve the situation in a good way. Ultimately, everyone agreed the game lost for the program whose operator made the error.

Two games played by SHREDDER suffered from network issues: game 3.3/18, GRIDGINKGOSHREDDER and g5.3/27, KOMODO-SHREDDER. SHREDDER was playing the game on machines in the 
cloud and at certain moments, Stefan Meyer-Kahlen had trouble reaching these machines. In round five, a repair left the game drawn but in round three, this was not the case.

Given the extra prize for the runner-up, CHESSBASE14 donated by Chessbase, Sonneborn-Berger scoring was used to determine positions 2, 3 and 4. Second went to SHREDDER, third to CHIRON and fourth to the combative GRIDGINKGO which had however been commendably draw-shy and scored twice as many wins. Just off the pace set by these three, JONNY placed fifth.

Table 2

The games of WSCC $2018^{2}$ with some FrITZ14 evaluations.

\begin{tabular}{|c|c|c|c|c|c|c|c|c|c|c|c|c|}
\hline \# & Rnd & Wh. & Bl. & \#m & Res. & ECO & Opening & Fgen & $7 \mathrm{~m}$ & $6 \mathrm{~m}$ & $5 \mathbf{m}$ & Endgame notes \\
\hline 01 & 1.1 & $\mathrm{C}$ & $\mathrm{K}$ & 58 & $1 / 2-1 / 2$ & D35 & Queen's Gambit Declined & $33 \mathrm{~b}$ & - & - & - & Clear draw at $33 \mathrm{~b},-0.38 \mathrm{~d} 28$ \\
\hline 02 & 1.2 & $\mathrm{~K}$ & $\mathrm{C}$ & 76 & $1-0$ & D04 & Queen's Pawn Game & - & - & - & - & C lost on time \\
\hline 03 & 1.3 & $\mathrm{C}$ & K & 29 & $1 / 2-1 / 2$ & D35 & Queen's Gambit Declined & - & - & - & - & \\
\hline 04 & 1.1 & G & $\mathrm{J}$ & 55 & $1 / 2-1 / 2$ & C50 & Giuco Piano & $51 \mathrm{~b}$ & $54 \mathrm{w}$ & $54 \mathrm{~b}$ & - & 54w/54b, n-man 'EGT' draw \\
\hline 05 & 1.2 & G & $\mathrm{J}$ & 91 & $1 / 2-1 / 2$ & C67 & Ruy Lopez & $58 w$ & $86 b$ & $91 \mathrm{w}$ & $91 b$ & 86b/91w/91b, EGT draw \\
\hline 06 & 1.3 & $\mathrm{~J}$ & $\mathrm{G}$ & 32 & $0-1$ & E90 & King's Indian & - & - & - & - & \\
\hline 07 & 2.1 & $\mathrm{~K}$ & $\mathrm{G}$ & 64 & $1-0$ & B90 & Sicilian, Najdorf & - & - & - & - & $64 \mathrm{~b},-0.02 \mathrm{~d} 27$ : lost by operator error \\
\hline 08 & 2.2 & $\mathrm{G}$ & K & 51 & $0-1$ & B13 & Caro-Kann defence, Exchange var. & $51 \mathrm{~b}$ & - & - & - & $51 \mathrm{~b},-8.26 \mathrm{~d} 23$ \\
\hline 09 & 2.3 & $\mathrm{~K}$ & G & 71 & $1-0$ & D70 & Neo-Grunfeld Defense & - & - & - & - & \\
\hline 10 & 2.1 & $\mathrm{~S}$ & $\mathrm{C}$ & 39 & $1 / 2-1 / 2$ & D37 & Queen's Gambit Declined & $38 \mathrm{~b}$ & - & - & - & Clear draw at $38 \mathrm{~b}, 0.00 \mathrm{~d} 22$ \\
\hline 11 & 2.2 & $\mathrm{C}$ & $\mathrm{S}$ & 70 & $1-0$ & B48 & Sicilian, Taimanov var. & - & - & - & - & CHIRON mated SHREDDER \\
\hline 12 & 2.3 & $\mathrm{~S}$ & $\mathrm{C}$ & 38 & $1 / 2-1 / 2$ & D37 & Queen's Gambit Declined & - & - & - & - & \\
\hline 13 & 3.1 & $\mathrm{~J}$ & K & 50 & $0-1$ & E11 & Bogo-Indian Defense & - & - & - & - & \\
\hline 14 & 3.2 & $\mathrm{~J}$ & K & 65 & $0-1$ & E11 & Bogo-Indian Defense & - & - & - & - & \\
\hline 15 & 3.3 & K & $\mathrm{J}$ & 48 & $1 / 2-1 / 2$ & D02 & Queen's Pawn Game & $28 w$ & - & - & - & Clear draw at $28 \mathrm{w}, 0.08 \mathrm{~d} 23$ \\
\hline 16 & 3.1 & $\mathrm{G}$ & $\mathrm{S}$ & 66 & $1-0$ & B85 & Sicilian, Scheveningen, Classical & - & - & - & - & 66w: White mates in $4 \mathrm{~m}$ \\
\hline 17 & 3.2 & $\mathrm{~S}$ & G & 85 & $1-0$ & E90 & King's Indian & - & $80 w$ & $80 \mathrm{~b}$ & $84 w$ & $80 \mathrm{w}: \mathrm{KBBPKBB}, d t m=17 \mathrm{~m}$ \\
\hline 18 & 3.3 & G & $\mathrm{S}$ & 60 & $1-0$ & D43 & Queen's Gambit Declined, semi-Slav & $59 b$ & - & - & - & $59 \mathrm{~b},+12.31 \mathrm{~d} 24:$ 'cloud' issues intervened \\
\hline 19 & 4.1 & $\mathrm{~S}$ & $\mathrm{~J}$ & 56 & $1 / 2-1 / 2$ & D45 & Queen's Gambit Declined, semi-Slav & - & $55 \mathrm{~b}$ & $56 w$ & - & $55 \mathrm{~b} / 56 \mathrm{w}$, EGT draw \\
\hline 20 & 4.2 & $\mathrm{~S}$ & $\mathrm{~J}$ & 80 & $1-0$ & D45 & Queen's Gambit Declined, semi-Slav & - & - & - & - & \\
\hline 21 & 4.3 & $\mathrm{~J}$ & $\mathrm{~S}$ & 61 & $1 / 2-1 / 2$ & D43 & Queen's Gambit Declined, semi-Slav & - & $61 b$ & $62 w$ & - & $61 \mathrm{~b} / 62 \mathrm{w}$, EGT draw \\
\hline 22 & 4.1 & $\mathrm{G}$ & $\mathrm{C}$ & 34 & $1 / 2-1 / 2$ & D31 & Queen's Gambit Declined & - & - & - & - & \\
\hline 23 & 4.2 & $\mathrm{C}$ & $\mathrm{G}$ & 101 & $1-0$ & E99 & King's Indian & - & $101 \mathrm{w}$ & - & - & EGT draw at $101 \mathrm{w}$ : lost by operator error \\
\hline 24 & 4.3 & $\mathrm{G}$ & $\mathrm{C}$ & 20 & $0-1$ & B90 & Sicilian, Najdorf & - & - & - & - & \\
\hline 25 & 5.1 & $\mathrm{~K}$ & $\mathrm{~S}$ & 56 & $1 / 2-1 / 2$ & A06 & Reti Opening & - & - & - & - & \\
\hline 26 & 5.2 & $S$ & K & 52 & $1 / 2-1 / 2$ & A88 & Dutch, Leningrad, main var. with c6 & - & $49 b$ & $51 \mathrm{~b}$ & $52 w$ & $49 \mathrm{~b} / 51 \mathrm{~b} / 52 \mathrm{w}$, EGT draw \\
\hline 27 & 5.3 & $\mathrm{~K}$ & $\mathrm{~S}$ & 48 & $1 / 2-1 / 2$ & B48 & Sicilian, Taimanov var. & $28 b$ & $46 w$ & $48 w$ & - & $28 \mathrm{~b},-0.09 \mathrm{~d} 26: 46 \mathrm{w} / 48 \mathrm{w}$, EGT draw \\
\hline 28 & 5.1 & $\mathrm{~J}$ & $\mathrm{C}$ & 60 & $1-0$ & E05 & Catalan, Open, Classical line & - & - & - & - & \\
\hline 29 & 5.2 & $\mathrm{~J}$ & $\mathrm{C}$ & 66 & $1 / 2-1 / 2$ & E05 & Catalan, Open, Classical line & $40 \mathrm{~b}$ & $64 w$ & $65 b$ & & 40b, 0.37d23: 64w/65b, EGT draw \\
\hline 30 & 5.3 & $\mathrm{C}$ & $\mathrm{J}$ & 71 & $1 / 2-1 / 2$ & D12 & Queen's Gambit Declined Slav & $68 w$ & & & & Clear draw at $68 \mathrm{w}, 0.00 \mathrm{~d} 24$ \\
\hline
\end{tabular}

Table 3 and 4

WSCC 2017 round by round, results and progress

\begin{tabular}{|c|c|c|c|c|c|c|c|}
\hline id & Program & r1 & r2 & r3 & r4 & r5 & Score \\
\hline K & KOMODO & C: $1 / 211 / 2$ & G: 111 & $\mathrm{~J}: \begin{array}{lll}1 & 1 & 1 / 2\end{array}$ & & S: $1 / 21 / 21 / 2$ & 9 \\
\hline $\mathrm{S}$ & SHREDDER & & C: $1 / 201 / 2$ & G: 010 & $\mathrm{~J}: 1 / 211 / 2$ & K: $1 / 21 / 21 / 2$ & $51 / 2$ \\
\hline $\mathrm{C}$ & CHIRON & $\mathrm{K}: 1 / 201 / 2$ & S: $1 / 21^{1 / 2}$ & & G: $1 / 210$ & J: $01 \frac{1}{2} 1 / 2$ & $51 / 2$ \\
\hline G & GRIDGINKGO & $\mathrm{J}: 1 / 21 / 21$ & K: 000 & S: 101 & C: $1 / 201$ & & $51 / 2$ \\
\hline J & JONNY & $\mathrm{G}: 1 / 21 / 20$ & & 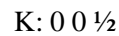 & $S: 1 / 20^{1 / 2}$ & C: $1_{1 / 2}^{1 / 2}$ & $41 / 2$ \\
\hline
\end{tabular}

\begin{tabular}{cccccc}
\hline Progress ... & r1 & r2 & r3 & r4 & r5 \\
\hline K & 2 & 5 & $71 / 2$ & & 9 \\
S & & 1 & 2 & 4 & $51 / 2$ \\
C & 1 & 3 & & $41 / 2$ & $51 / 2$ \\
G & 2 & 2 & 4 & $51 / 2$ & \\
J & 1 & & $1 \frac{1}{2}$ & $2 \frac{1}{2} 2$ & $41 / 2$ \\
\hline
\end{tabular}

After the 30 games, average length $58 \mathrm{~m}$ and $50 \%$ won, the clear winner was KOMODO with 9 points from 15. It retained its 2017 title after distancing the field early, courtesy of a 3-0 against GRIDGINKGO

\footnotetext{
${ }^{2}$ Fgen notes when the game first has one piece at most per side, allowing FINALGEN (Romero, 2018) to attempt a definitive evaluation, in theory if not in practice. ' $7 \mathrm{~m}$ ', ' $6 \mathrm{~m}$ ' and ' $5 \mathrm{~m}$ ' refer to the number of men when the position can be consulted in 'EGT' endgame tables (Bleicher, 2018; de Man et al., 2018; Lomonosov team, 2018; Tamplin, 2018).
} 
in round 2 and a $2 \frac{1}{2}-1 / 2$ against JONNY in round 3, both being unique achievements. The ICGA warmly congratulates KOMODO on a fine win and thanks all participants for the action-packed contest.

Table 5

The WSCC 2017 cross-table

\begin{tabular}{|c|c|c|c|c|c|c|c|c|c|c|c|}
\hline id & Program & $\mathbf{K}$ & $\mathbf{S}$ & C & G & $\mathbf{J}$ & W & D & $\mathbf{L}$ & Score & S-B \\
\hline $\mathrm{K}$ & KOMODO & & $11 / 2: 1 / 21 / 21 / 2$ & $2: 1 / 211 / 2$ & $3: 111$ & $21 / 2: 11 \frac{1 / 2}{2}$ & 6 & 6 & 0 & 9 & \\
\hline $\mathrm{S}$ & SHREDDER & $11 / 2: 1 / 21 / 2^{1 / 2}$ & & $1: 1 / 201 / 2$ & 1: 010 & 2: $1 / 21 \frac{1}{1 / 2}$ & 2 & 7 & 3 & $5 \frac{1}{2}$ & $33^{1 / 2}$ \\
\hline $\mathrm{C}$ & CHIRON & 1: $1 / 201 / 2$ & $2: 1 / 211 / 2$ & & $11 / 2: 1 / 210$ & 1: $0^{1 / 2} 2^{1 / 2}$ & 2 & 7 & 3 & $5 \frac{1}{2}$ & $323 / 4$ \\
\hline G & GRIDGINKGO & 0: 0000 & 2: 101 & 11/2: $1 / 201$ & & $2: 1 / 21 / 21$ & 4 & 3 & 5 & $5 \frac{1}{2}$ & $281 / 4$ \\
\hline $\mathrm{J}$ & JONNY & 1/2: $00 \begin{array}{lll}1 / 2 & \end{array}$ & $1: 1 / 201 / 2$ & $2: 1^{1 / 2} 1 / 2$ & $1: 1 / 21 / 20$ & & 1 & 7 & 4 & $41 / 2$ & \\
\hline
\end{tabular}

\section{REFERENCES}

Bleicher, E. (2018). https://tinyurl.com/icga002. Bleicher interface to Nalimov-format DTC/M/Z sub7-man EGTs.

CPW (2018). https://tinyurl.com/icga046. Resited biographies of programs, authors and operators.

de Man, R., Fiekas, N. and Guo, B. (2018). https://tinyurl.com/icga007. Fiekas' interface to 'Syzygy formatted' de Man sub-7-man and Guo 7-man DTZ ${ }_{50}$ " EGTs.

Krabbenbos, J., van den Herik, H.J. and Haworth, G.Mㄷ. (2018a). WCSC 2018: the $8^{\text {th }}$ World Chess Software Championship. http://centaur.reading.ac.uk/78437/. ICGA Journal, 40(3), \$\$-\$. doi:\$\$.

Krabbenbos, J., van den Herik, H.J. and Haworth, G.McC. (2018b). WCCC 2018: the $24^{\text {th }}$ World Computer Chess Championship. http://centaur.reading.ac.uk/78436/. ICGA Journal, 40(3), \$\$-\$\$. doi:\$\$.

Krabbenbos, J., van den Herik, H.J. and Haworth, G.MC. (2018c). WSCC 2018: the 2018 World Speed Computer Chess Championship. http://centaur.reading.ac.uk/78438/. This note plus an annotated pgn file of the games. ICGA Journal, 40(3), \$\$-\$ doi:\$\$.

Lomonosov team (2018) https://tinyurl.com/icga008. 7-man DTM EGT query service.

Romero, P.P. (2018). https://tinyurl.com/icga013. FINALGEN information and download.

Tamplin, J. (2018). https://tinyurl.com/icga006. Interface to sub-6-mand and some 6-man EGTs. 